\title{
A note on moment-based sufficient dimension reduction estimators
}

\author{
YuexiaO Dong
}

The two main groups of moment-based sufficient dimension reduction methods are the estimators for the central space and the estimators for the central mean space. The former group includes methods such as sliced inverse regression, sliced average variance estimation and sliced average third-moment estimation, while ordinary least squares and principal Hessian directions belong to the latter group. We provide unified frameworks for each group of estimators in this short note. The central space estimators can be unified as inverse conditional cumulants, while Stein's Lemma is used to motivate the central mean space estimators.

Keywords And PHRASES: Central mean space, Central space, Conditional cumulants, Stein's Lemma.

\section{INTRODUCTION}

Suppose $X$ is a $p$ dimensional predictor and $Y$ is a univariate response. Sufficient dimension reduction (SDR) $(\mathrm{Li}$, 1991; Cook, 1994) aims to find subspace $\mathcal{S}$ such that

$$
Y \Perp X \mid P_{\mathcal{S}} X,
$$

where $\Perp$ means statistical independence, and $P_{(\cdot)}$ denotes the projection operator with respect to the standard inner product. Under mild conditions (Yin, Li and Cook, 2008), the intersection of all $\mathcal{S}$ satisfying (1) still satisfies (1). We call this intersection the central space of regressing $Y$ on $X$ and denote it by $\mathcal{S}_{Y \mid X}$. In the case when the focus is on the regression mean $E(Y \mid X)$, sufficient dimension reduction targets at finding the subspace $\mathcal{S}$ satisfying

$$
E(Y \mid X) \Perp Y \mid P_{\mathcal{S}} X .
$$

When the intersection of all $\mathcal{S}$ satisfying (2) still satisfies (2), we call it the central mean space (Cook and Li, 2002) and denote it by $\mathcal{S}_{E(Y \mid X)}$.

Let $\mu=E(X)$ and $\Sigma=\operatorname{Cov}(X)$. Then $Z=\Sigma^{-1 / 2}(X-\mu)$ denotes the standardized predictor. Due to the invariance property of the central space (Cook, 1998) and a similar property for the central mean space, we have $\mathcal{S}_{Y \mid X}=$ $\Sigma^{-1 / 2} \mathcal{S}_{Y \mid Z}$ and $\mathcal{S}_{E(Y \mid X)}=\Sigma^{-1 / 2} \mathcal{S}_{E(Y \mid Z)}$. Hence one can identify the $Z$-scale central space or the $Z$-scale central mean space, and then transform them back to the $X$-scale spaces.
Without loss of generality, we work with the standardized predictor, and assume throughout this paper that $E(X)=0$ and $\operatorname{Var}(X)=I$.

Two of the most popular central space estimators are sliced inverse regression (SIR; Li, 1991) and sliced average variance estimator (SAVE; Cook and Weisberg, 1991). To find additional structure in regression mixtures, Yin and Cook (2003) proposed sliced average third-moment estimation (SAT). By re-expressing these moments-based methods in terms of inverse conditional cumulants, we cast them under one unified framework. A nice byproduct is that the seemingly complicated SAT estimator is revealed to have a simple form as inverse conditional third-order $\mathrm{cu}-$ mulant.

Our second contribution is to provide a unified treatment of moment-based central mean space estimators through Stein's Lemma. While it is well-known that first-order and second-order Stein's Lemmas lead to ordinary least squares estimator (OLS; Li and Duan, 1989) and principal Hessian directions estimator (PHD; Li, 1992) respectively, we demonstrate that third-order Stein's Lemma can be used to motivate the illusive central mean space estimator proposed in Yin and Cook (2004).

The rest of the paper is organized as follows. We study inverse conditional cumulants for central space estimators in Section 2. We use Stein's Lemma to motivate central mean space estimators in Section 3. We conclude the paper with some discussions in Section 4.

\section{INVERSE CONDITIONAL CUMULANTS AND ESTIMATORS OF THE CENTRAL SPACE}

\subsection{Review of estimators in $\mathcal{S}_{Y \mid X}$}

Suppose $\beta$ is a basis of $\mathcal{S}_{Y \mid X}$ and denote $\operatorname{Span}(\beta)$ as the column space of $\beta$. Let $P_{\beta}=\beta\left(\beta^{T} \beta\right)^{-1} \beta^{T}$ be the projection matrix onto $\operatorname{Span}(\beta)$ and $Q_{\beta}=I-P_{\beta}$ be the projection onto the orthogonal complement of $\operatorname{Span}(\beta)$. For the ease of reference, we state the following conditions commonly seen in the sufficient dimension reduction literature.

C1. $E\left(X \mid \beta^{T} X\right)=P_{\beta} X$

C2. $\operatorname{Var}\left(X \mid \beta^{T} X\right)=Q_{\beta}$;

C3. $E\left(A \otimes A A^{T} \mid \beta^{T} X\right)=0$, where $A=X-E\left(X \mid \beta^{T} X\right)$. 
Here $\otimes$ in $\mathrm{C} 3$ denotes the Kronecker product. All three conditions are satisfied if the marginal distribution of $X$ is normal.

The next proposition summarizes the common estimators in the central space, and we state it without proof. Please refer to Yin and Cook (2003) for details.

Proposition 1. Suppose $\beta$ is a basis of $\mathcal{S}_{Y \mid X}$.

1. If $C 1$ is true, then $E(X \mid Y) \subseteq \mathcal{S}_{Y \mid X}$.

2. If $C 1$ and $C 2$ are true, then $\operatorname{Span}\{I-\operatorname{Var}(X \mid Y)\} \subseteq$ $\mathcal{S}_{Y \mid X}$.

3. If $C 1, C 2$ and $C 3$ are true, then $\operatorname{Span}\left\{E^{T}(B \otimes\right.$ $\left.\left.B B^{T} \mid Y\right)\right\} \subseteq \mathcal{S}_{Y \mid X}$, where $B=X-E(X \mid Y)$.

The results above lead to the SIR estimator in $\mathrm{Li}$ (1991), the SAVE estimator in Cook and Weisberg (1991), and the SAT estimator in Yin and Cook (2003) respectively. Specifically, let $\mathcal{M}_{\mathrm{SIR}}=\operatorname{Var}\{E(X \mid Y)\}, \mathcal{M}_{\mathrm{SAVE}}=E[\{I-$ $\left.\operatorname{Var}(X \mid Y)\}^{2}\right]$ and $\mathcal{M}_{\mathrm{SAT}}=E\left\{E^{T}\left(B \otimes B B^{T} \mid Y\right) E(B \otimes\right.$ $\left.\left.B B^{T} \mid Y\right)\right\}$. Then the column space of $\mathcal{M}_{\mathrm{SIR}}, \mathcal{M}_{\mathrm{SAVE}}$ and $\mathcal{M}_{\mathrm{SAT}}$ can all be used to recover the central space $\mathcal{S}_{Y \mid X}$.

\subsection{Inverse conditional cumulants}

We review the definition of unconditional cumulants first. Recall that $X=\left(X_{1}, X_{2}, \ldots, X_{p}\right)^{T} \in \mathbb{R}^{p}$ is a random vector. Denote $\mu_{r}=E\left(X_{r}\right)$ as components of the mean vector $E(X), \mu_{r s}=E\left(X_{r} X_{s}\right)$ as components of the second moment matrix, and $\mu_{r s t}=E\left(X_{r} X_{s} X_{t}\right)$ as the third moments. For $\xi=\left(\xi_{1}, \ldots, \xi_{p}\right)^{T}$, the Taylor expansion of the moment generating function $M_{X}(\xi)=E\left\{\exp \left(\xi^{T} X\right)\right\}$ is

$$
\begin{gathered}
M_{X}(\xi)=1+\sum_{r=1}^{p} \xi_{r} \mu_{r}+\frac{1}{2 !} \sum_{r, s=1}^{p} \xi_{r} \xi_{s} \mu_{r s} \\
+\frac{1}{3 !} \sum_{r, s, t=1}^{p} \xi_{r} \xi_{s} \xi_{t} \mu_{r s t}+\cdots .
\end{gathered}
$$

Consider the Taylor expansion of $\log M_{X}(\xi)$ as follows,

$$
\begin{gathered}
\log M_{X}(\xi)=\sum_{r=1}^{p} \xi_{r} \kappa_{r}+\frac{1}{2 !} \sum_{r, s=1}^{p} \xi_{r} \xi_{s} \kappa_{r s} \\
+\frac{1}{3 !} \sum_{r, s, t=1}^{p} \xi_{r} \xi_{s} \xi_{t} \kappa_{r s t}+\cdots
\end{gathered}
$$

The cumulants are then defined as the coefficients $\kappa_{r}, \kappa_{r s}$ and $\kappa_{r s t}$ in (4). By comparing (3) and (4), one can follow McCullagh (1987) and establish the relationship between the first few moments and cumulants as

$$
\begin{aligned}
\kappa_{r} & =\mu_{r}, \\
\kappa_{r s} & =\mu_{r s}-\mu_{r} \mu_{s}, \\
\kappa_{r s t} & =\mu_{r s t}-\mu_{r s} \mu_{t}-\mu_{r t} \mu_{s}-\mu_{s t} \mu_{r}+2 \mu_{r} \mu_{s} \mu_{t} .
\end{aligned}
$$

Conditional cumulants can be defined similarly. First denote inverse conditional moments $\mu_{r}^{X \mid Y}=E\left(X_{r} \mid Y\right), \mu_{r s}^{X \mid Y}=$
$E\left(X_{r} X_{s} \mid Y\right)$, and $\mu_{r s t}^{X \mid Y}=E\left(X_{r} X_{s} X_{t} \mid Y\right)$. Parallel to (5), we define inverse conditional cumulants as combinations of inverse conditional moments

$$
\begin{aligned}
& \kappa_{r}^{X \mid Y}=\mu_{r}^{X \mid Y}, \\
& \kappa_{r s}^{X \mid Y}=\mu_{r s}^{X \mid Y}-\mu_{r}^{X \mid Y} \mu_{s}^{X \mid Y}, \\
& \kappa_{r s t}^{X \mid Y}=\mu_{r s t}^{X \mid Y}-\mu_{r s}^{X \mid Y} \mu_{t}^{X \mid Y}-\mu_{r t}^{X \mid Y} \mu_{s}^{X \mid Y} \\
& \quad-\mu_{s t}^{X \mid Y} \mu_{r}^{X \mid Y}+2 \mu_{r}^{X \mid Y} \mu_{s}^{X \mid Y} \mu_{t}^{X \mid Y} .
\end{aligned}
$$

Recall that $\mu_{r}^{X \mid Y}, \mu_{r s}^{X \mid Y}$ and $\mu_{r s t}^{X \mid Y}$ are essential components of $E(X \mid Y), \operatorname{Var}(X \mid Y)$ and $E\left(B \otimes B B^{T} \mid Y\right)$ in Proposition 1. We now construct $\mathcal{K}_{1}(X \mid Y) \in \mathbb{R}^{p}, \mathcal{K}_{2}(X \mid Y) \in \mathbb{R}^{p \times p}$ and $\mathcal{K}_{3}(X \mid Y) \in \mathbb{R}^{p \times p \times p}$ as follows. Let the $r^{t h}$ component of $\mathcal{K}_{1}(X \mid Y)$ be $\kappa_{r}^{X \mid Y}$, the $(r, s)^{t h}$ component of $\mathcal{K}_{2}(X \mid Y)$ be $\kappa_{r s}^{X \mid Y}$, and the $(r, s, t)^{t h}$ component $\mathcal{K}_{3}(X \mid Y)$ be $\kappa_{r s t}^{X \mid Y}$.

In a parallel fashion, we can replace $Y$ in the definitions above with $\beta^{T} X$ and define $\mathcal{K}_{1}\left(X \mid \beta^{T} X\right), \mathcal{K}_{2}\left(X \mid \beta^{T} X\right)$ and $\mathcal{K}_{3}\left(X \mid \beta^{T} X\right)$. Before we present our main result, we state conditions $\mathrm{C} 1, \mathrm{C} 2$ and $\mathrm{C} 3$ in their equivalent forms

$$
\begin{aligned}
& \mathrm{C} 1^{\prime} . \mathcal{K}_{1}\left(X \mid \beta^{T} X\right)=P_{\beta} X ; \\
& \mathrm{C} 2^{\prime} . \mathcal{K}_{2}\left(X \mid \beta^{T} X\right)=Q_{\beta} ; \\
& \mathrm{C} 3^{\prime} . \mathcal{K}_{3}\left(X \mid \beta^{T} X\right)=0 .
\end{aligned}
$$

For the ease of presentation, we also rearrange the thirdorder inverse cumulant array $\mathcal{K}_{3}(X \mid Y)$ as a $p^{2} \times p$ matrix, with the $(t-1) p+s^{t h}$ column of $\mathcal{K}_{3}^{T}(X \mid Y)$ being

$$
\begin{aligned}
& \mathcal{K}_{3}^{s t}(X \mid Y)=E\left(X X_{s} X_{t} \mid Y\right)-E\left(X X_{s} \mid Y\right) E\left(X_{t} \mid Y\right) \\
& \quad-E\left(X X_{t} \mid Y\right) E\left(X_{s} \mid Y\right)-E\left(X_{s} X_{t} \mid Y\right) E(X \mid Y) \\
& \quad+2 E(X \mid Y) E\left(X_{s} \mid Y\right) E\left(X_{t} \mid Y\right) .
\end{aligned}
$$

In another word, $\mathcal{K}_{3}^{s t}(X \mid Y)=\left(\kappa_{1 s t}^{X \mid Y}, \ldots, \kappa_{p s t}^{X \mid Y}\right)^{T}$ for $s, t=$ $1,2, \ldots, p$. Similarly, we rearrange the array $\mathcal{K}_{3}\left(X \mid \beta^{T} X\right)$ as a $p^{2} \times p$ matrix. We now present SIR, SAVE and SAT as inverse conditional cumulants.

Proposition 2. Suppose $\beta$ is a basis of $\mathcal{S}_{Y \mid X}$.

1. If $C 1^{\prime}$ is true, then $\mathcal{K}_{1}(X \mid Y) \subseteq \mathcal{S}_{Y \mid X}$.

2. If $C 1^{\prime}$ and $C 2^{\prime}$ are true, then $\operatorname{Span}\left\{I-\mathcal{K}_{2}(X \mid Y)\right\} \subseteq$ $\mathcal{S}_{Y \mid X}$.

3. If $C 1^{\prime}, C 2^{\prime}$ and $C 3^{\prime}$ are true, then $\operatorname{Span}\left\{\mathcal{K}_{3}^{T}(X \mid Y)\right\} \subseteq$ $\mathcal{S}_{Y \mid X}$.

Proof. From (6), the definition of $\mathcal{K}_{1}(X \mid Y)$ and the definition of $\mathcal{K}_{2}(X \mid Y)$, we know $\mathcal{K}_{1}(X \mid Y)=E(X \mid Y)$ and $\mathcal{K}_{2}(X \mid Y)=E\left(X X^{T} \mid Y\right)-E(X \mid Y) E^{T}(X \mid Y)=\operatorname{Var}(X \mid Y)$. After replacing $Y$ with $\beta^{T} X$, we see immediately that $C 1$ is equivalent to $C 1^{\prime}$, and $C 2$ is equivalent to $C 2^{\prime}$. The first two parts of Proposition 2 then follow directly from the first two parts of Proposition 1.

For part 3, denote $\mathcal{M}_{B}=E\left(B \otimes B B^{T} \mid Y\right)$ with $B=$ $X-E(X \mid Y)$. From the definition of the Kronecker product, the $(t-1) p+s^{t h}$ column of $\mathcal{M}_{B}^{T}$ is 


$$
\begin{aligned}
\mathcal{M}_{B}^{s t}= & E\left[\{X-E(X \mid Y)\}\left\{X_{s}-E\left(X_{s} \mid Y\right)\right\}\right. \\
& \left.\left\{X_{t}-E\left(X_{t} \mid Y\right)\right\} \mid Y\right] .
\end{aligned}
$$

Compare (8) with the definition of $\mathcal{K}_{3}^{s t}(X \mid Y)$ in (7). By noticing equalities such as

$$
\begin{aligned}
& E\left\{X X_{s} E\left(X_{t} \mid Y\right) \mid Y\right\}=E\left(X X_{s} \mid Y\right) E\left(X_{t} \mid Y\right) \text { and } \\
& E\left\{X E\left(X_{s} \mid Y\right) E\left(X_{t} \mid Y\right) \mid Y\right\}=E(X \mid Y) E\left(X_{s} \mid Y\right) E\left(X_{t} \mid Y\right),
\end{aligned}
$$

it is easy to see that $\mathcal{M}_{B}^{s t}=\mathcal{K}_{3}^{s t}(X \mid Y)$. It follows that $\mathcal{M}_{B}=$ $\mathcal{K}_{3}(X \mid Y)$. Denote $\mathcal{M}_{A}=E\left(A \otimes A A^{T} \mid \beta^{T} X\right)$ with $A=X-$ $E\left(X \mid \beta^{T} X\right)$. We can use a similar argument to show $\mathcal{M}_{A}=$ $\mathcal{K}_{3}\left(X \mid \beta^{T} X\right)$, which guarantees the equivalence between $C 3$ and $C 3^{\prime}$. Part 3 of Proposition 2 then follows from the last part of Proposition 1.

The insight of Proposition 2 is that SIR, SAVE and SAT can be viewed as inverse conditional cumulants. In the case of SAT, condition $\mathrm{C} 3$ can be replaced with a simpler condition $\mathcal{K}_{3}\left(X \mid \beta^{T} X\right)=0$, and its kernel matrix $E\left(B \otimes B B^{T} \mid Y\right)$ with $B=X-E(X \mid Y)$ becomes just the third-order inverse conditional cumulant array $\mathcal{K}_{3}(X \mid Y)$.

\section{STEIN'S LEMMA AND ESTIMATORS OF THE CENTRAL MEAN SPACE}

\subsection{Review of estimators in $\mathcal{S}_{E(Y \mid X)}$}

In the seminal paper of $\mathrm{Li}$ and Duan (1989), it was demonstrated that without knowing the underlying link function $f(\cdot)$, the classical ordinary least squares (OLS) estimator can recover the index $\beta$ in the single-index model $Y=f\left(\beta^{T} X\right)+\epsilon$ correctly up to a constant multiplier. Here $\epsilon$ is the random error independent of $X$. In the modern language of Cook and Li (2002), we say that the OLS estimator belongs to the central mean space $\mathcal{S}_{E(Y \mid X)}$. The principal Hessian directions estimator in $\mathrm{Li}$ (1992), as well as the estimator in Yin and Cook (2004), also belong to the central mean space. Let $\operatorname{vec}(I)$ be the vectorization of the $p \times p$ identity matrix $I$. Denote $\mathcal{M}_{\mathrm{OLS}}=\mathcal{M}_{O} \mathcal{M}_{O}^{T}, \mathcal{M}_{\mathrm{PHD}}=\mathcal{M}_{P} \mathcal{M}_{P}^{T}$ and $\mathcal{M}_{\mathrm{YC}}=\mathcal{M}_{Y}^{T} \mathcal{M}_{Y}$, where

$$
\begin{aligned}
& \mathcal{M}_{O}=E(X Y), \\
& \mathcal{M}_{P}=E\left[\{Y-E(Y)\} X X^{T}\right] \text { and } \\
& \mathcal{M}_{Y}=E\left[\{Y-E(Y)\} X \otimes X X^{T}\right]-E(Y X) \otimes I \\
& \quad-I \otimes E(Y X)-\operatorname{vec}(I) E^{T}(Y X) .
\end{aligned}
$$

We summarize these estimators in the next result without proof. Please refer to Yin and Cook (2004) for details. Note that in Section 2.1, the conditions C1, C2 and C3 are assumed to be true for the basis of the central space. In the next proposition, we assume conditions $\mathrm{C} 1, \mathrm{C} 2$ and $\mathrm{C} 3$ to be true for the basis of the central mean space.

Proposition 3. Suppose $\beta$ is a basis of $\mathcal{S}_{E(Y \mid X)}$.

1. If $C 1$ is true, then $\operatorname{Span}\left(\mathcal{M}_{\mathrm{OLS}}\right) \subseteq \mathcal{S}_{E(Y \mid X)}$.

2. If $C 1$ and $C 2$ are true, then $\operatorname{Span}\left(\mathcal{M}_{\mathrm{PHD}}\right) \subseteq \mathcal{S}_{E(Y \mid X)}$.
3. If $C 1, C 2$ and $C 3$ are true, then $\operatorname{Span}\left(\mathcal{M}_{\mathrm{YC}}\right) \subseteq$ $\mathcal{S}_{E(Y \mid X)}$.

Although the OLS and PHD estimators have relatively simple forms, the estimator $\mathcal{M}_{\mathrm{YC}}$ is not easy to come up with. The original derivation in Yin and Cook (2004) actually starts from the SAT estimator, and then gets $\mathcal{M}_{\mathrm{YC}}$ after carefully delineating the components of SAT that belong to the central space. Just as we have seen in Section 2.2 that the third-order central space estimator SAT can be unified with the first-order method SIR and the second-order method SAVE, we will cast central mean space estimators $\mathcal{M}_{\mathrm{OLS}}, \mathcal{M}_{\mathrm{PHD}}$ and $\mathcal{M}_{\mathrm{YC}}$ in one unified framework.

\subsection{Stein's Lemma}

Recall that for $X=\left(X_{1}, X_{2}, \ldots, X_{p}\right)^{T}$, we have assumed that $E(X)=0$ and $\operatorname{Var}(X)=I$ from the outset. To motivate central mean space estimators via Stein's Lemma, we further assume in this section that $X_{i} \sim N(0,1)$ for $i=1,2, \ldots, p$ and $X_{i} \Perp X_{j}$ for $i \neq j$. In the famous paper of Stein (1981), it is demonstrated that for any function $g\left(X_{i}\right)$ with first through third order derivatives denoted by $g^{\prime}\left(X_{i}\right)$, $g^{\prime \prime}\left(X_{i}\right)$ and $g^{\prime \prime \prime}\left(X_{i}\right)$, we have

$$
\begin{aligned}
E\left\{g^{\prime}\left(X_{i}\right)\right\} & =E\left\{X_{i} g\left(X_{i}\right)\right\}, \\
E\left\{g^{\prime \prime}\left(X_{i}\right)\right\} & =E\left\{\left(X_{i}^{2}-1\right) g\left(X_{i}\right)\right\}, \text { and } \\
E\left\{g^{\prime \prime \prime}\left(X_{i}\right)\right\} & =E\left\{\left(X_{i}^{3}-3 X_{i}\right) g\left(X_{i}\right)\right\} .
\end{aligned}
$$

Consider $g(X)=E(Y \mid X)$ as a function of $X$. Denote partial derivatives $d_{r}=\partial g(X) / \partial X_{r}, d_{r s}=\partial^{2} g(X) / \partial X_{r} \partial X_{s}$ and $d_{r s t}=\partial^{3} g(X) / \partial X_{r} \partial X_{s} \partial X_{t}$. We construct $\mathcal{D}_{1} \in \mathbb{R}^{p}$, $\mathcal{D}_{2} \in \mathbb{R}^{p \times p}$ and $\mathcal{D}_{3} \in \mathbb{R}^{p \times p \times p}$ as follows. Let the $r^{\text {th }}$ component of $\mathcal{D}_{1}$ be $E\left(d_{r}\right)$, the $(r, s)^{t h}$ component of $\mathcal{D}_{2}$ be $E\left(d_{r s}\right)$, and the $(r, s, t)^{t h}$ component $\mathcal{D}_{3}$ be $E\left(d_{r s t}\right)$. For the ease of presentation, we rearrange the third-order derivatives array $\mathcal{D}_{3}$ as a $p^{2} \times p$ matrix, where the entry in the $r^{t h}$ column and $(t-1) p+s^{t h}$ row is $E\left(d_{r s t}\right)$.

The next result combines central mean space estimators $\mathcal{M}_{\mathrm{OLS}}, \mathcal{M}_{\mathrm{PHD}}$ and $\mathcal{M}_{\mathrm{YC}}$ in one unified framework.

Proposition 4. Suppose $X \sim N(0, I)$.

1. $\mathcal{D}_{1}=\mathcal{M}_{O}$.

2. $\mathcal{D}_{2}=\mathcal{M}_{P}$.

3. $\mathcal{D}_{3}=\mathcal{M}_{Y}$.

4. $\operatorname{Span}\left(\mathcal{D}_{1}\right) \subseteq \mathcal{S}_{E(Y \mid X)}, \quad \operatorname{Span}\left(\mathcal{D}_{2}\right) \subseteq \mathcal{S}_{E(Y \mid X)}$ and $\operatorname{Span}\left(\mathcal{D}_{3}^{T}\right) \subseteq \mathcal{S}_{E(Y \mid X)}$.

Proof. From the first equation in (10), we know $E\left\{\partial E(Y \mid X) / \partial X_{r}\right\}=E\left\{X_{r} E(Y \mid X)\right\}=E\left(X_{r} Y\right)$. The lefthand side and the right-hand side are the $r^{t h}$ entry of $\mathcal{D}_{1}$ and $\mathcal{M}_{O}$ respectively. Thus we have $\mathcal{D}_{1}=\mathcal{M}_{O}$.

To find the $(r, s)^{t h}$ component of $\mathcal{D}_{2}$, we need to treat the two cases $r \neq s$ and $r=s$ separately. If $r \neq s$, we have

$$
\begin{aligned}
& E\left\{\partial^{2} E(Y \mid X) / \partial X_{r} \partial X_{s}\right\}=E\left[X_{r}\left\{\partial E(Y \mid X) / \partial X_{s}\right\}\right] \\
= & E\left\{X_{r} X_{s} E(Y \mid X)\right\}=E\left(X_{r} X_{s} Y\right) \\
= & E\left[\{Y-E(Y)\} X_{r} X_{s}\right],
\end{aligned}
$$


where the last equality is true because $E\left(X_{r} X_{s}\right)=0$. If $r=s$, we use the second equation in (10) and get

$$
\begin{aligned}
& E\left\{\partial^{2} E(Y \mid X) / \partial X_{r}^{2}\right\}=E\left\{\left(X_{r}^{2}-1\right) E(Y \mid X)\right\} \\
= & E\left\{\left(X_{r}^{2}-1\right) Y\right\}=E\left[\{Y-E(Y)\} X_{r}^{2}\right],
\end{aligned}
$$

where the last equality is true because $E\left(X_{r}^{2}\right)=1$. Together with the definition of $\mathcal{M}_{P}$ in (9), we have $\mathcal{D}_{2}=\mathcal{M}_{P}$.

To find the $(r, s, t)^{t h}$ component of $\mathcal{D}_{3}$, there are three separate cases. When $r, s$ and $t$ are all distinct, we have

$$
E\left\{\partial^{3} E(Y \mid X) / \partial X_{r} \partial X_{s} \partial X_{t}\right\}=E\left(X_{r} X_{s} X_{t} Y\right) .
$$

When two of $r, s$ and $t$ are the same, we assume without loss of generality that $r=s \neq t$. Then we have

$$
E\left\{\partial^{3} E(Y \mid X) / \partial X_{r}^{2} \partial X_{t}\right\}=E\left\{\left(X_{r}^{2}-1\right) X_{t} Y\right\} .
$$

When $r=s=t$, we use the third equation in (10) and get

$$
E\left\{\partial^{3} E(Y \mid X) / \partial X_{r}^{3}\right\}=E\left\{\left(X_{r}^{3}-3 X_{r}\right) Y\right\} .
$$

Now we turn to $\mathcal{M}_{Y}=E\left[\{Y-E(Y)\} X \otimes X X^{T}\right]-$ $E(Y X) \otimes I-I \otimes E(Y X)-\operatorname{vec}(I) E^{T}(Y X) \in \mathbb{R}^{p^{2} \times p}$ defined in (9), and denote its entry in the $r^{\text {th }}$ column and $(t-1) p+s^{t h}$ row as $\mathcal{M}_{Y}(r, s, t)$. For the ease of presentation, we view $\mathcal{M}_{Y}$ as a $p \times p \times p$ array, where the $(r, s, t)^{t h}$ entry is $\mathcal{M}_{Y}(r, s, t)$.

When $r, s$ and $t$ are all distinct, the last three terms in the definition of $\mathcal{M}_{Y}$ all become 0 , and the $(r, s, t)^{t h}$ component of $\mathcal{M}_{Y}$ becomes $E\left[\{Y-E(Y)\} X_{r} X_{s} X_{t}\right]$, which matches the right-hand side of (11) as $E\left(X_{r} X_{s} X_{t}\right)=0$. When $r=s=t$, all four terms in the definition of $\mathcal{M}_{Y}$ remain. The $(r, s, t)^{t h}$ component of $\mathcal{M}_{Y}$ becomes $E\left[\{Y-E(Y)\} X_{r}^{3}\right]-3 E\left(Y X_{r}\right)$, which matches the right-hand side of (13) by noting that $E\left(X_{r}^{3}\right)=0$. When two of $r, s$ and $t$ are the same, only one of the last three terms in the definition of $\mathcal{M}_{Y}$ remains. For instance, if we have $r=s \neq t$, the $(r, s, t)^{t h}$ component of $\mathcal{M}_{Y}$ becomes $E\left[\{Y-E(Y)\} X_{r}^{2} X_{t}\right]-E\left(Y X_{t}\right)$, which matches the right-hand side of (12) because $E\left(X_{r}^{2} X_{t}\right)=0$. Together we have shown $\mathcal{D}_{3}=\mathcal{M}_{Y}$.

It remains to prove part 4 . Let $\beta$ be a basis of $\mathcal{S}_{E(Y \mid X)}$. From the definition of the central mean space in (2), we know $E(Y \mid X)=E\left(Y \mid \beta^{T} X\right)$. As a result,

$$
\frac{\partial E(Y \mid X)}{\partial X}=\frac{\partial E\left(Y \mid \beta^{T} X\right)}{\partial X}=\frac{\partial \beta^{T} X}{\partial X} \frac{\partial E\left(Y \mid \beta^{T} X\right)}{\partial \beta^{T} X} .
$$

Because $\partial \beta^{T} X / \partial X=\beta$, we have $\operatorname{Span}\left(\mathcal{D}_{1}\right)=$ $\operatorname{Span}(E\{\partial E(Y \mid X) / \partial X\}) \subseteq \operatorname{Span}(\beta)=\mathcal{S}_{E(Y \mid X)}$. Following similar arguments, we have $\operatorname{Span}\left(\mathcal{D}_{2}\right) \subseteq \mathcal{S}_{E(Y \mid X)}$ and $\operatorname{Span}\left(\mathcal{D}_{3}^{T}\right) \subseteq \mathcal{S}_{E(Y \mid X)}$

Although we assume normality to facilitate the connections between the central mean space estimators, we have seen in Proposition 3 that the normality assumption can be replaced with weaker conditions $\mathrm{C} 1, \mathrm{C} 2$ and $\mathrm{C} 3$. The insight of Proposition 4 is that just as OLS and PHD can be motivated from first and second-order partial derivatives of $E(Y \mid X)$, we can use the third-order partial derivatives of $E(Y \mid X)$ to construct the much more complicated estimator in Yin and Cook (2004).

\section{DISCUSSIONS}

We provide a general treatment of moment-based sufficient dimension reduction estimators in this note. On one hand, popular central space estimators such as SIR, SAVE and SAT are cast under the unified framework of inverse conditional cumulants. On the other hand, Stein's Lemma is used to motivate central mean space estimators such as OLS, PHD and the estimator in Yin and Cook (2004). Although the focus of the paper is to reveal connections between existing moment-based estimators, we provide possibilities to come up with new sufficient dimension reduction estimators. For example, one can follow the principle of Proposition 4, and construct new estimators in the central mean space based on fourth-order partial derivatives of $E(Y \mid X)$.

Stein's Lemma is not limited to motivate estimators in the central mean space. Actually one can construct a general family of sufficient dimension reduction estimators through Stein's Lemma. Instead of choosing $g(X)$ to be $E(Y \mid X)$, we can set $g(X)=E\left(Y^{k} \mid X\right)$ for any integer $k$ and apply Stein's Lemma. This will lead to the central $k$ th moment space estimators studied in Yin and Cook (2002). An interesting case here is that when we choose $g(X)=E\left(e^{i Y} \mid X\right)$ and apply Stein's Lemma, we will get estimators in the central space (Zhu, Zhu and Wen, 2010).

\section{Received 20 March 2014}

\section{REFERENCES}

Cook, R. D. (1994). On the interpretation of regression plots. Journal of the American Statistical Association 89, 177-189. MR1266295

Cook, R. D. (1998). Regression Graphics. New York: Wiley. MR1645673

Cook, R. D. and LI, B. (2002). Dimension reduction for conditional mean in regression. The Annals of Statistics 30, 455-474. MR1902895

Cook, R. D. and Weisberg, S. (1991). Discussion of "Sliced inverse regression for dimension reduction". Journal of the American Statistical Association 86, 316-342. MR1137117

LI, K. C. (1991). Sliced inverse regression for dimension reduction (with discussion). Journal of the American Statistical Association 86, 316-342. MR1137117

LI, K. C. (1992). On principal Hessian directions for data visualization and dimension reduction: another application of Stein's Lemma. Journal of the American Statistical Association 87, 1025-1039. MR1209564

LI, K. C. and DuAn, N. (1989). Regression analysis under link violation. Annals of Statistics 17, 1009-1052. MR1015136

McCullagh, P. (1987). Tensor Methods in Statistics. London: Chapman and Hall. MR0907286

Stein, C. (1981). Estimation of the mean of a multivariate normal distribution. The Annals of Statistics 9, 1135-1151. MR0630098

YIN, X. and Cook, R. D. (2002). Dimension reduction for the conditional kth moment in regression. Journal of the Royal Statistical Society Ser. B 64, 159-175. MR1904698 
YIN, X. and CoOK, R. D. (2003). Estimating the central subspace via inverse third moment. Biometrika 90, 113-125. MR1966554

YIN, X. and Cook, R. D. (2004). Dimension reduction via marginal fourth moments in regression. Journal of Computational and Graphical Statistics 13, 554-570. MR2087714

YIN, X., LI, B. and CoOK, R. D. (2008). Successive direction extraction for estimating the central subspace in a multipleindex regression. Journal of Multivariate Analysis 99, 1733-1757. MR2444817
Zhu, L. P., Zhu, L. X. and Wen, S. (2010). On dimension reduction in regressions with multivariate response. Statistica Sinica 20, 12911307. MR2730185

Yuexiao Dong

Temple University

Philadelphia, PA, 19122

USA

E-mail address: ydong@temple.edu 